\title{
BMJ Open Experiences of a non-clinical set of adolescents and young adults living with persistent pain: a qualitative metasynthesis
}

\author{
Liv Fegran (1) , ${ }^{1,2}$ Berit Johannessen, ${ }^{1}$ Mette Spliid Ludvigsen (i) , ${ }^{3,4}$ \\ Thomas Westergren, ${ }^{1}$ Magnhild Høie (i) , ${ }^{5}$ Åshild Slettebø, ${ }^{5}$ Gudrun Rohde, ${ }^{1,6}$ \\ Sølvi Helseth, ${ }^{7}$ Kristin Haraldstad ${ }^{1}$
}

To cite: Fegran L,

Johannessen B, Ludvigsen MS, et al. Experiences of a nonclinical set of adolescents and young adults living with persistent pain: a qualitative metasynthesis. BMJ Open 2021;11:e043776. doi:10.1136/ bmjopen-2020-043776

- Prepublication history for this paper is available online. To view these files, please visit the journal online (http://dx.doi. org/10.1136/bmjopen-2020043776).

Received 13 August 2020 Revised 16 March 2021 Accepted 28 March 2021
Check for updates

\section{(C) Author(s) (or their} employer(s)) 2021. Re-use permitted under CC BY-NC. No commercial re-use. See rights and permissions. Published by BMJ.

For numbered affiliations see end of article.

Correspondence to

Dr Liv Fegran; liv.fegran@uia.no

\section{ABSTRACT}

Purpose Around $15 \%-30 \%$ of adolescents and young adults (AYAs) experience persistent or chronic pain. The purpose of this study was to synthesise evidence from qualitative primary studies on how AYAs in a non-clinical population experience living with persistent pain.

Method A qualitative metasynthesis guided by Sandelowski and Barroso's guidelines was used. The databases Medline, Embase, Cinahl, PsycINF0, Mednar and ProQuest were searched for studies from 1 January 2005 to 15 February 2021. Inclusion criteria were AYAs aged 13-24 years with first-hand experience of living with persistent, recurrent or episodic non-clinical pain in any body site. Pain associated with a medical diagnosis, malignant diseases, medical procedures or sport activities was excluded.

Results 0 2618 screened records, data from nine studies conducted in a Western cultural context including 184 participants (127 female and 57 male aged 11-28 years) were analysed into metasummaries and a metasynthesis. Headaches was the most focused pain condition $(n=5)$, while three of the studies did not specify type of pain. The participants' experiences were characterised by (1) juggling pain with everyday life; (2) exploring sources of information to manage pain; (3) AYAs' use of medication to find relief and (4) non-pharmacological strategies for pain relief.

Conclusion These AYAs experience of how pain influences everyday life, and their striving to find relief from pain by support from family, friends, professionals and the Internet should be strongly respected. Public health nurses and other healthcare professionals encountering AYAs need to respect their pain experiences, and to support them in healthy coping strategies. Further studies on this issue are needed, especially research focusing on AYAs pain in exposed populations and AYAs from non-Western cultures.

\section{INTRODUCTION}

In recent years, it has been recognised that pain comprises a common and growing health problem among adolescents and young adults (AYAs). The prevalence varies in non-clinical populations defined as a population without a clear clinical diagnosis, or not recruited from a clinical context as, for example, a pain
Strengths and limitations of this study

- The use of a robust and transparent review method revealed that, despite a vast amount of research on this topic, individual adolescents and young adults (AYAs') experiences are difficult to identify and extract.

- The qualitative approach is an important contribution to this research field by providing a deeper understanding of why and how AYAs strive to find relief for their pain.

- Despite the challenge of identifying AYAs' voices, synthesising data from a sample of 184 AYAs from a non-clinical setting has contributed to a novel understanding of their experiences when living with persistent pain.

- The lack of studies providing data from minority cultures, exposed populations or studies from countries outside North America or Europe is a limitation.

- Exclusion of studies describing clinical pain, procedure pain and pain in connection with sports could possibly have concealed aspects of pain experiences relevant to understand the complexity of AYAs pain.

clinic. However, research has shown a trend towards a high and increasing prevalence of persistent or chronic pain, varying from $20 \%$ to $35 \%$ in different studies. ${ }^{1-6}$ Such pain can lead to significant interference in daily functioning and daily life, and is associated with poor sleep, stress, impaired school/college functioning and absenteeism. ${ }^{7-10}$ Studies also show that more female than male AYAs report pain; moreover, living with pain affects female AYAs more negatively. ${ }^{411} 12$ Further, pain problems can negatively influence a young person's quality of life and health. ${ }^{4913}$ Experiencing pain in adolescence is related to pain problems later in adulthood, and research indicates that pain problems might also have serious long-term consequences. ${ }^{14-16}$

Subjectivity is at the core of the experience of pain as reflected in various definitions. 
McCaffery's well-known definition of pain often cited in nursing studies describes pain as: 'Pain is whatever the experiencing person says it is, existing whenever he says it does'. ${ }^{17}$ According to International Association for the Study of Pain (IASP) nomenclature, pain is always subjective, and the IASP definition recognises the central role of emotions in defining pain as 'an unpleasant sensory and emotional experience associated with, or resembling that associated with, actual or potential tissue damage"'(Merskey, p.24) ${ }^{18}$ These definitions highlight the importance of accepting the person's perception and experience of pain. Pain is a complex phenomenon and can be a symptom of underlying problems reflecting several psychological, social and physical factors. ${ }^{4} 1920$ Adolescence is a vulnerable period in life between childhood and adulthood, and can be challenging with respect to developmental tasks, independence from caregivers, social development and peer relationships. ${ }^{21} 22$ However, this period of development also provides an opportunity for young people to develop positive health behaviours to improve their functioning and to prevent future painrelated problems. ${ }^{523} 24$

Previous studies have given us knowledge of the prevalence of pain and its associated factors; however, pain among adolescents is still an understudied topic, especially in non-clinical populations. Few qualitative studies explore adolescents' personal experiences of living with pain, how they cope with it, and how it can influence their emotional and physical functioning. Research suggests that they use methods familiar to them to manage their pain.$^{25}{ }^{26}$ Differences in coping might be related to each adolescent's personality and coping style. ${ }^{27}$ In qualitative studies of young people with chronic pain, different coping strategies were identified, and coping strategies were shown to be influenced by family, friends and attitudes to pain. ${ }^{28} 29$

Some qualitative studies have shown that female AYAs are more likely to talk to friends about pain problems than are male AYAs, and to use pain medication as a coping strategy. ${ }^{30}$ Moreover, many parents were unaware of their adolescent's pain, and the adolescents did not speak to their parents about their pain experience. ${ }^{313} \mathrm{~A}$ Swedish study of adolescents and their use of over-thecounter (OTC) medications revealed that OTC drug use was significantly influenced by parents and peers. ${ }^{31}$ Studies from Norway have shown that adolescents have a high frequency of use of OTC medications and use them as a coping strategy. ${ }^{28} 29$

To be able to help adolescents to cope with pain and develop prevention strategies, it is important to improve our understanding of adolescents' experience with pain, and how they cope with the problem throughout adulthood.

\section{Aim}

The aim of this study was to identify and synthesise evidence from qualitative primary studies on how AYAs in a non-clinical population experience living with persistent pain. A protocol describing the design of this study was published in the Joanna Briggs Institute Database of Systematic Reviews \& Implementation Reports in $2014 .^{32}$

\section{METHODS}

The metasynthesis was guided by Sandelowski and Barroso's guidelines for synthesising qualitative research from published qualitative primary research studies, ${ }^{33}$ and based on the Preferred Reporting Items for Systematic Reviews and Meta-Analyses guidelines for systematic reviews. ${ }^{34}$ The metasynthesis process consisted of five steps: (1) formulating the purpose and rationale of the study; (2) searching for and retrieving relevant qualitative research reports; (3) critically appraising the included studies; (4) classifying the findings and (5) synthesising the findings.

\section{Search strategy}

Our search strategy aimed to find both published and unpublished studies (Grey literature), and a four-step search strategy was developed by three of the authors (LF, $\mathrm{KH}$ and MSL). The electronic databases Medline (OVID), Embase (OVID), Cinahl (OVID), PsycINFO (OVID) and Proquest were searched initially in February 2017 using Medical Subject Heading and index term combinations of the keywords adolescents OR young adults AND pain AND qualitative studies. Second, after identifying relevant keywords (table 1 ), the search strategy was modified to fit each database in collaboration with a librarian, and the final search was conducted on 15 February 2021. Limiting age and language was the easy part; however, defining our search strategy concerning 'pain' was

\begin{tabular}{|c|c|c|}
\hline Population & Phenomenon of interest & Context \\
\hline $\begin{array}{l}\text { adolescent }{ }^{\star} \text { OR teenager } \\
\text { OR young adult* OR young } \\
\text { people* OR young person* OR } \\
\text { youngster }^{\star} \text { OR youth*}\end{array}$ & $\begin{array}{l}\text { abdominal pain* OR back pain* OR backache* } \\
\text { OR face pain* OR facial pain* OR headache* OR } \\
\text { jaw pain* OR limb pain* OR low back pain* OR } \\
\text { musculoskeletal pain* OR myofascial pain* OR neck } \\
\text { pain* OR shoulder pain* OR tension headache* OR } \\
\text { widespread pain* OR chronic pain* OR persistent } \\
\text { pain* OR recurrent pain* OR everyday pain* }\end{array}$ & $\begin{array}{l}\text { experience* OR comprehension* } \\
\text { OR attitude* OR emotion* OR view* } \\
\text { OR experience }{ }^{\star} \text { OR opinion* OR } \\
\text { perception* OR belie* OR feeling* OR } \\
\text { know* OR understand }^{*} \text { OR adaptation* }\end{array}$ \\
\hline
\end{tabular}




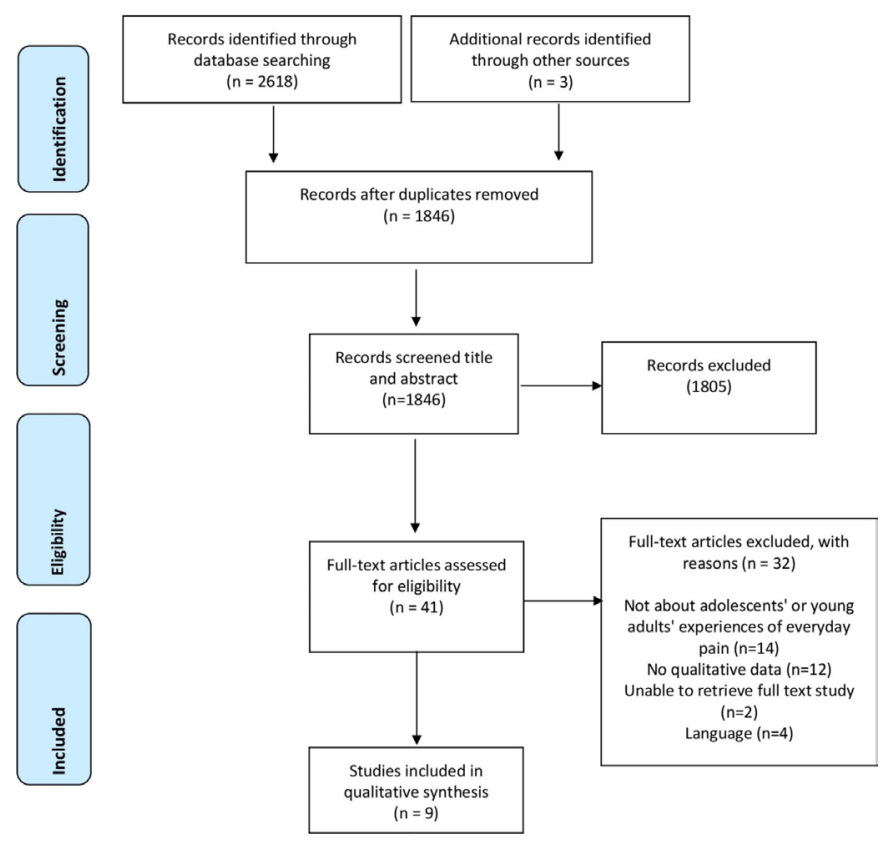

Figure 1 The inclusion process.

challenging. Using the unspecific term 'pain' produced an overwhelming number of hits. Therefore, in collaboration with the librarian we decided to narrow the search to more specific types of pain described in previous relevant studies. The database search strategy with its search blocks is presented in table 1 .

The third step was to search the reference lists of the included studies, and finally the publications citing the included studies were checked for eligibility through the 'find citing articles' in OVID (PsycINFO, Nursing, Medline), ISI Web of Science, Scopus, Cinahl and Google Scholar. The flow diagram (figure 1) reflects all steps in the inclusion process.

\section{Inclusion criteria}

This review aimed to consider studies published in English, Norwegian, Swedish or Danish describing a non-clinical population of adolescents (13-18 years) and young adults (19-24 years) with first-hand experiences of living with persistent pain not associated with cancer or similar life-threatening malignant diseases or clinical diagnosis, regardless of gender, ethnicity or country of origin, and length of years in living with everyday pain.

\section{Exclusion criteria}

During the screening process, many studies focusing on specific non-clinical pain conditions were identified. According to the aim of this study, we consequently added the following exclusion criteria: pain in connection with medical procedures, cerebral palsy, sport activities, surgery, medication testing, treatment testing and instrument validation. Publications involving migraine headache or chronic tension-type headache were considered a clinical diagnosis and were therefore excluded. ${ }^{35} 36$

\section{Selecting and appraising studies for the metasynthesis}

Four of the authors (LF, MSL, GR and KH) screened titles and abstracts and appraised relevant reports for inclusion according to the aim of the study and the inclusion/exclusion criteria. Any discrepancy between the researchers about whether a study should be included or not was discussed until consensus was reached. Of the 2618 records screened, nine were eligible for inclusion in the metasynthesis.

\section{Quality appraisal}

The studies relevant for inclusion were independently critically appraised by two of the authors (LF and MSL) according to the Joanna Briggs Critical Appraisal Checklist for Interpretive and Critical Research. ${ }^{37}$ Congruency between aims, methodology, data collection and analysis methods was evaluated. Overall, the congruency between philosophical perspectives, methodologies and methods used was strong. The exceptions were two studies with an unclear congruity between philosophical perspective and methodology. ${ }^{39}$ The most poorly addressed issue was the influence of the researcher on the research and vice versa, and five of the nine studies included did not address this issue. ${ }^{38}{ }^{40-43}$ The results of the critical appraisal are presented in table 2.

\section{Analysis}

In line with Sandelowski and Barroso's handbook, two approaches to qualitative research synthesis were used: qualitative metasummaries and qualitative metasyntheses. ${ }^{33}$ Qualitative metasummaries are quantitative aggregations of qualitative findings from the included studies presented in table 3. Qualitative metasyntheses are new interpretations of target findings from primary studies. The text from the results sections was considered the studies' target findings consisting of quotations from AYAs, or the primary researchers' interpretation of AYAs' own experiences. The authors' presentation of results from other studies, presentation of analytical procedures or discussion of findings were considered as non-target findings. ${ }^{33}$

After extraction, the target findings were imported into the data management software programme NVivo V.11 for further analysis. ${ }^{44} \mathrm{~A}$ phenomenological-hermeneutic analysis inspired by Lindseth and Norberg ${ }^{45}$ was performed by three of the reviewers (LF, BJ and $\mathrm{KH}$ ). The text was initially read line-by-line to identify meaning units. ${ }^{46}$ These were further condensed and abstracted to form main themes and subthemes. The authors discussed the analysis until consensus, and finally, a comprehensive understanding of AYAs' experiences of living with persistent pain was described under three main themes.

\section{PATIENT AND PUBLIC INVOLVEMENT}

This systematic review is based on published primary studies and does not involve public involvement. 


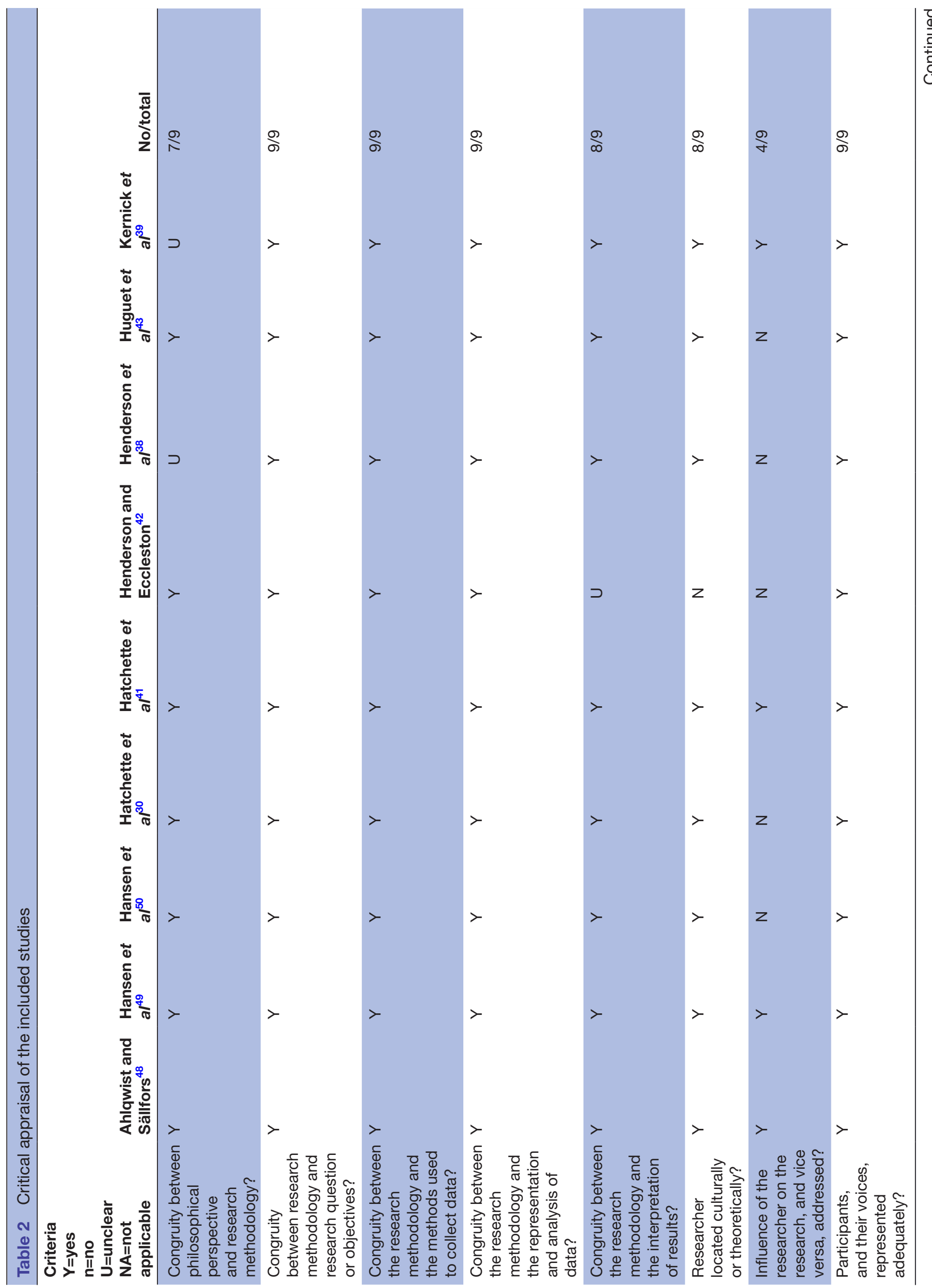

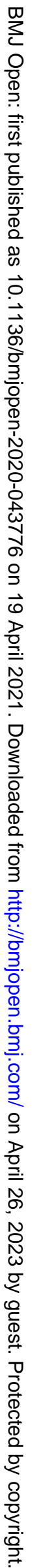




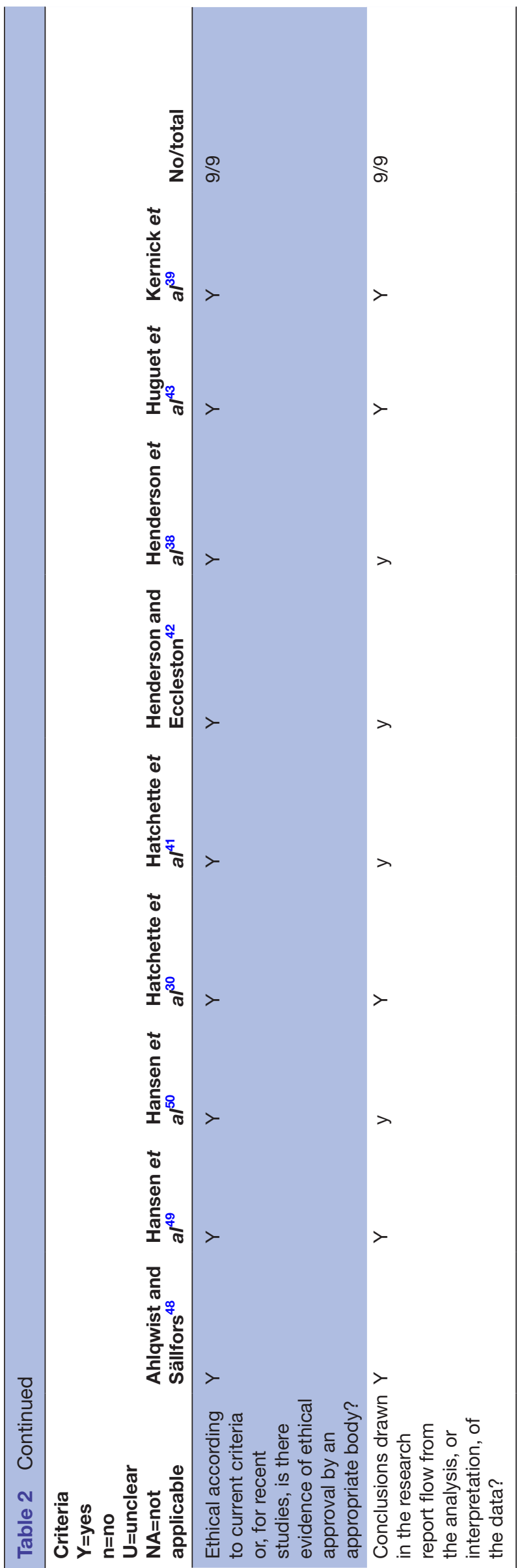

RESULTS

The findings are presented as metasummaries supported by tables and figures, and as a metasynthesis presented under three themes.

\section{Metasummaries}

In five of the nine studies, ${ }^{30} 38-434849$ headache was the focused pain condition, and one study ${ }^{42}$ included participants with various pain conditions in addition to headache. In the remaining three studies type of pain was not described. ${ }^{3048}$ Due to the low number of studies in the topic, we decided to include one study with participants aged in the lower age range (11-13 years) ${ }^{39}$ All the studies were conducted in a Western cultural context, with three in Canada ${ }^{304143}$ and six in Europe (UK, Denmark and Sweden). ${ }^{38} 394248-50$ Eight of the studies had a specific focus on the pain management of AYAs, while one study focused only on their experiences of living with pain. ${ }^{39}$ There was a preponderance of female participants $(127 / 184)$. Characteristics of the included studies are presented in table 3 .

\section{Metasynthesis}

Qualitative metasyntheses offer novel interpretations of the target findings from primary studies. ${ }^{33}$ The three main themes characterising AYAs' experiences of living with everyday pain were: (1) juggling pain with everyday life; (2) exploring sources of information to manage everyday pain; (3) AYAs' use of medication to find relief and (4) non-pharmacological strategies for pain relief. These and related subthemes will be elaborated in the following text.

\section{Main theme 1: juggling pain with everyday life}

Five of the included studies provided data for this main theme. ${ }^{3039434849}$ Living with pain strongly influenced the participants' daily lives regarding education, social life and other activities. ${ }^{39} 4349$

Education appeared to be demanding for AYAs and they were easily left behind and had to drop out of courses because of absence. ${ }^{43}$ Pain related to stress was often connected to demanding situations in school such as examinations. ${ }^{49}$ Participants also described how their teachers could be reluctant to accept the impact of headache on their school performance, ${ }^{30}$ as expressed by one adolescent: 'Teachers think that you just want to get out of class.' Despite struggling for acknowledgement of their own pain, AYAs expressed negative attitudes towards peers' doubts that their pain expressions were real: 'Well the real pain is pain that everyone would feel. But 'wussy' pain is something that one person would whine about and the other person would just sort of live with it.'

Some of the participants had lived with their pain condition for a long time and were reluctant to believe that they could get rid of it: 'I've had them since I was so little I don't think there is much I can do.' Despite enduring pain, some of the participants even belittled the idea that their pain problem was serious enough to get 


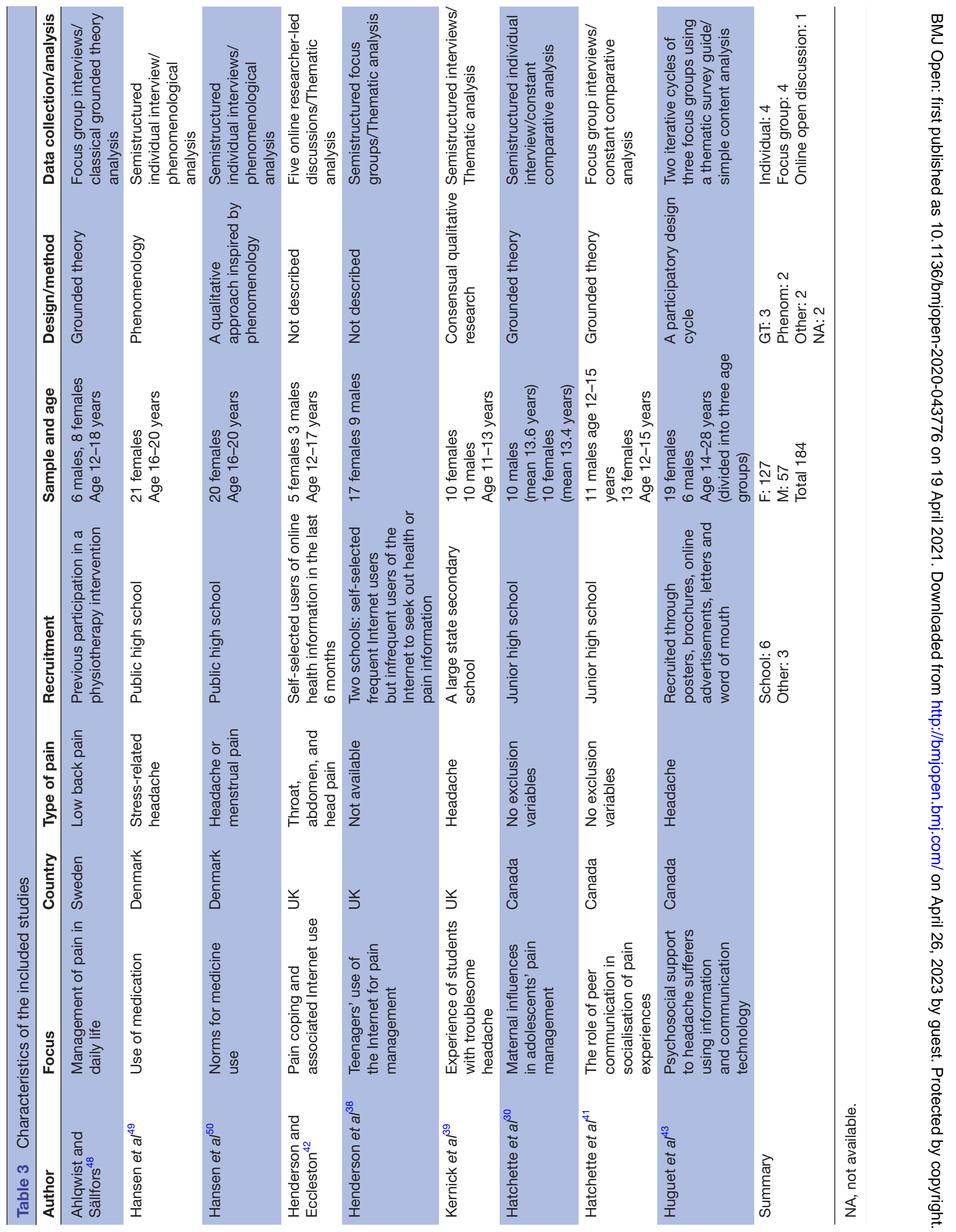


attention $^{30}$ : 'Haven't asked for help because headache is not serious, it's just a headache.'

Ahlqwist and Sällfors ${ }^{48}$ noted that the participants described how pain-free moments had a great impact on their coping and could put them more in control of their lives:

I changed my way of thinking and I am now more open with myself as well as with others. My mood has improved, and I feel that I want so much more than I did before. I have more energy and I understand myself better, and thinking in a different way even helps me to face other types of problems.

Their sleep quality and social life increased and they reached a turning point toward a more normalised life:

I got much better. And I think it was a real boost for me to feel that after the past twelve weeks I no longer had any pain. So, it was that easy. That was the end of it somehow. And since then I haven't had any more problems with it. I feel I'm on a good path.

\section{Main theme 2: exploring sources of information to manage} everyday pain

Five of the included studies provided data for this main theme. $^{38} 39424348$ They revealed that AYAs living with enduring pain strived to gather information from different sources, hoping to find answers to their pain problems. AYAs' information-seeking behaviour was influenced by the transition process from being a child to becoming an independent young adult; for some, their family's norms strongly influenced their perceptions of pain and pain management. ${ }^{39} 40$ The AYAs expressed a strong desire to be involved in their own pain management. They had a need to be informed about their pain condition and which relevant management strategies to use, in addition to social support. ${ }^{43}$ The older the participants were, the more the family norms were substituted by perceptions of what was common, accepted, and expected by friends and peers. ${ }^{30} 40$ They seemed to position themselves by attaining similar attitudes to the other group members: 'They just take a painkiller, of course, like me, right. If they have a headache, they just take a painkiller.'

The AYAs expressed a strong desire to receive information about pain management, and information from professionals helping to strengthen their independence. ${ }^{3948}$ Besides having adults who told them what to do, the AYAs also experienced that being involved in finding solutions to their pain problems made them feel valued. The school health service staff played an important role in taking their pain seriously and in supporting them:

But I think that's exactly what it was, a positive experience. That it was even available, that there was a place where you could go to see someone who was trained and could help you. And then to be able to agree on what the problem is and what to do about it. And even in some way, it wasn't just up to me, but like a joint effort. Yeah, and like someone who cared.

Seeking information through the media and the Internet appeared to be a way for AYAs to learn more about their pain condition, to be able to make informed decisions, and to handle their pain problems independently. The Internet was used intensively for checking symptoms before talking to others about them ${ }^{38} 4248$ :

I think it varies in what condition it is ... because, like say if it's something that you don't want anyone else to know about or something like that, then you'll go and try and sort it out yourself [by using the Internet] rather than tell someone.

Information from the Internet was mainly used in addition to other information sources such as parents and professionals: 'Mainly I would use the Internet as a source of supplementary information on top of any info that I get from my mum.' AYAs also described how information on the Internet was experienced as unsuitable for their age or pain condition. ${ }^{38} 48$ Others talked about anxiety and worries when they found information describing worst-case scenarios, and not information suitable for their condition:

I just had a cold or something and then it was like, are you having trouble breathing? And obviously I was, yeah, I'm congested so I do. And then straight away it was phone an ambulance, phone an ambulance when... and so it's, it's not detailed enough... And they have the whole list of things. At the start it's just like 'you've got a common cold', and then at the end it's like 'you're having a heartattack woooooo, spooky.

Comparing multiple Internet sources on the same subject was also used to validate information:

I would crosscheck any info which I would find with the info on other websites to see if it's true. Also, I would look to see if the sites had cited any sources of info, as this usually suggests reliability.

The information on the Internet appeared to be less reliable than information from other sources, so the AYAs felt a need to validate it:

Because, like there are a lot of different things about, like on the Internet. Like some people just put like rubbish on the Internet but some people do actually like, like, know what they are talking about so it's like really what you click on.

The severity and endurance of their pain problem seemed to determine whether they had to seek additional professional help:

I think, if it was a minor pain, you would just let it wear off, whereas if it were a major one you probably wouldn't look online, you'd go and see your doctor or book an appointment with your doctor. 
Main theme 3: AYAs' use of medication to find relief

All of the nine included studies provided data for this main theme. AYAs' pain management was strongly influenced by their parents' or families' norms. ${ }^{38-41}$ The younger the participants were, the more they followed the rules and guidelines provided by their parents. ${ }^{40}$ The mothers were often in charge of the treatment of pain at home, and the use of OTC analgesics was common ${ }^{41}$ :

But if it's, like, [paracetamol] and stuff like that, they've never been particularly against it. They've been really, like, 'if you feel unwell, better to take a couple of [paracetamol] than feel ill.

After acquiring autonomy concerning the use of analgesics, some of the AYAs described how they deviated from the many expectations of others despite receiving approval.

AYAs' threshold to take medication varied greatly. ${ }^{49}$ Those who had low tolerance for pain had a similar low threshold for choosing analgesics to cope with pain. ${ }^{49}$ While some regarded pain as a normal part of everyday life, others strongly avoided pain by using analgesics to relieve it: 'So I'm pretty good at taking an [acetaminophen] or [ibuprofen] or something, if (.) I mean, if I just have the teeny tiniest bit of a headache, because I hate having a headache.' Hansen et al focused on AYAs' motives for analgesic use, and noted that they were often grounded in a combination of performance and participation-related functions ${ }^{49}$ :

And then just before, I thought, like, Oh no. This just cannot get spoiled... Now I'll take something and then I'll avoid getting a headache all day. If I just take five [acetaminophen] now, then nothing will come... It's just that if there was something I really wanted to do, then I was afraid there was something that would ruin it.

Performance was connected to being concentrated during school hours and managing homework in the evening; to do so, many participants used analgesics to keep up with both their own goals and their families' expectations:

I haven't taken medicine all my life. I think it was two years ago [starting high school], or something like that, where one really started. I mean if I, for example, had a headache, then I couldn't concentrate on my homework. And seeing as that's important, so that I can keep up in school, then one almost has to.

While many AYAs had increased their use of analgesics, others opposed this and demonstrated a different view:

...You don't take [paracetamol] at all? ... No, I try to avoid it. And they were really surprised, because I guess it's something they do, that's normal. And because it's normal, we don't talk about it. It's something one expects everyone does once in a while.
Umm. But then there's ones like me who don't do it so often."

Situations with low levels of stress were reported to reduce pain: 'If one is healthy in daily life, if one is less stressed and stuff like that, then it makes it so that one has less of a need for medicine.' In addition to reducing pain, some participants also used analgesics to deal with social anxiety, or to reduce the pressure in stressful situations:

I'm very good at it when I don't feel well, because I am so stressed every day and so then it's two [acetaminophen] or painkillers or something else that goes down fast, because I'm busy.

Even those who usually avoided taking pain medication regarded it as acceptable to do so to meet performance goals, and AYAs' expectations of being able to participate clearly influenced the use of analgesics. ${ }^{49}$ At home, when they were in more relaxed surroundings, they often used alternative strategies to manage pain, such as taking a rest or going to sleep. ${ }^{49}$ The availability of OTC medication influenced the use of analgesics: 'Because if you have a headache it's easier just to go to the Co-op and just go and buy paracetamol.'

Some of the participants talked about their friends' impulsive use of OTC medications:

Some people take too much medication. Some of my friends have (ibuprofen) and they just take it for everything. They don't wait and see if their headache goes away or wait and see if they're just overreacting or something. They just automatically take it.

Others were aware of the side effects of OTC medications and adjusted their use accordingly: 'When I feel like I'm getting a headache, I just take something... so then it's not going to get worse... I read the label to know the amount and how often to take them.'

Despite expressing concern about taking OTC analgesics, the participants also acknowledged the importance of using such medications if necessary: 'I'd rather have no pain for a little bit, than have to NOT take painkillers and have pain all the time.'

Non-pharmacological strategies for pain relief

In addition to analgesics, non-medical interventions were described as helpful tools to reduce pain. ${ }^{39} 404349$ Strategies providing AYAs with customisable and interactive coping tools, such as pain diaries, web-based information or tools to enable them to discuss their pain with peers, supported their ability to get a sense of control. ${ }^{43} 48$ Trusting their body's ability to control pain rather than having the pain control their lives was described as increasing their self-efficacy ${ }^{48}$ : 'I believe that my body can ... do many things. And I prove it too, I get rid of that headache every time, without taking anything.' Pain diaries were seen as useful tools to keep track of their pain and of factors that might be influencing it. Pain diaries could also support them in setting goals for themselves and in tracking their progress concerning pain and the 
development of coping strategies. Non-medical interventions could be an attitude developed by the AYAs themselves, or as a part of the inherited family practice. The most common non-medical intervention for headache was to drink a lot of water, in addition to strategies such as sleeping, relaxing, getting fresh air, doing leisure activities, or taking vitamins. 4849 Older AYAs tended to employ strategies such as using distractions, massage and exercise. ${ }^{43}$ Younger AYAs trusted their mothers' recommendations, while older AYAs behaved more according to the more-or-less tacit norms within their peer group. ${ }^{40}$ Alternative strategies to taking analgesics were discussed within their groups, and the participants reported how peers attempted to reduce their use of medication:

So it's often a debate. Because my friends are pretty observant about it. I've got a friend, T, who hates me for it. He can get so furious with me every time, 'Sit down and I'll make you some chamomile tea'... And then I say, 'No, T, I don't believe it works.' And then he goes crazy, because his opinion, he's $100 \%$ sure that natural medicine, it does the job just as well.

\section{DISCUSSION}

This metasynthesis including data from nine qualitative studies with 184 participating AYAs (127 female and 57 male) aims to give a voice to those AYA who struggle with persistent pain. To cope with their pain, AYAs had to juggle pain with their everyday life, explore various sources of information to manage their pain, and use both pharmacological and non-pharmacological strategies for pain relief.

Our findings indicate how living with pain strongly influences AYAs' everyday life, and how their pain experience is influenced by culture, friends and family. Some of the AYAs experienced doubts that their pain expressions were real, and that persistent pain was regarded as just an excuse to avoid certain situations. The newly revised definition of pain from the IASP emphasises the emotional aspect of pain, and the importance of believing in the person having pain and claims that 'a person's report of an experience of pain should be respected. ${ }^{51}$ The IASP's ${ }^{51}$ change from definition of pain as what a person says it is, to the definition of respecting a person's pain expression, could reflect our developing knowledge about the complexity of pain. This could be especially important in relation to AYAs with unspecific persistent pain.

The issue of not being believed could mirror a perception that today's young people may be less sturdy than previous generations, ${ }^{9} 52$ despite research revealing increasing pressure on today's adolescents both physically, socially and emotionally. ${ }^{53}$ Today's understanding of pain appears to be strongly influenced by defining it through its visible appearance, which undermines the complexity of enduring pain in a non-clinical adolescent population.
The AYAs experience of support from their school/ college health service staff could indicate that they acknowledged their pain, even if it did not present as a somatic problem. Teachers play an important role in children's and adolescents' coping processes ${ }^{954}$; however, some of the AYAs in our study experienced teachers who did not believe them when they talked about their pain. ${ }^{39}$ These findings are supported by studies showing that teachers tend to offer more support and empathy to an adolescent whose pain was related to a medical diagnosis than medically 'unexplained' pain. ${ }^{55}{ }^{56}$ Lack of understanding from teachers could be challenging, and be a part of the impaired education performance and high rates of school absenteeism described in research on AYAs with chronic pain. ${ }^{57}$ It is important to acknowledge AYAs' experiences, as emphasised in the revised pain definition from the IASP: 'a person's report of an experience as pain should be respected. ${ }^{51}$

Our findings indicate the extent of adolescents' search for answers from various sources to manage pain on their own. In a study from Australia, adolescents with pain were described to have little knowledge about pain and how to cope with it, and did not know who they could contact for help or information. ${ }^{55}$ According to our findings, the internet was one of the most intensively used sources for exploring pain and pain management, although the information accessed was not always deemed suitable to provide the answers needed, ${ }^{58}$ and AYAs sometimes found the information scary and inappropriate for their pain condition. These findings are in accordance with other studies that criticise information from the internet as not being individualised,$^{54}$ even if users' background is crucial in developing education programmes. ${ }^{59}$ Another issue to consider is that much information posted on the internet is not evidence based. A study on pain and pain management revealed that few web-based applications were scientifically sound, ${ }^{60}$ which could explain why information gleaned from the internet is often experienced as unsuitable, incorrect or exaggerated. The same question concerning reliability of information could apply to peers, who could have various reasons for sharing their opinions. Our findings show that it is important to be both equal to their peers, as well as to be able to oppose them. Both positions might strongly contribute to identity formation, and thus peers could be of great support to AYAs with pain. ${ }^{61}$ Healthcare professionals seemed to be the people AYAs trusted the most, because of their knowledge about pain and ability to balance this with their previous experience of helping AYAs to manage their pain. These findings are supported by Fegran et a ${ }^{62}$ who describe AYAs experiences of healthcare professionals' crucial role in caring for children and adolescents with chronic conditions during the transition to adolescence and adult life.

Some of the participants in our review expressed concerns about taking OTC analgesics to reduce stress and anxiety. However, they also acknowledged the importance of using medications if necessary. Pain management appeared to be strongly influenced by their parents' 
or families' norms, even if family attitudes as a source for managing pain gradually weakened as AYAs matured. These findings are in accordance with other studies showing that adolescents' primary source of information for using OTC analgesics is their parents, and that the mother's attitude toward using OTC analgesics influenced adolescent use. ${ }^{284163}$ The increasing use of OTC drugs is worrying because continuous use might prevent adolescents from learning healthier coping strategies. Instead of helping the adolescents to cope with pain, highfrequency use of OTC drugs could develop to become the start of lifelong use of medication. ${ }^{29}$

\section{Clinical implications}

Our findings might help increase healthcare professionals' knowledge of how to approach and support AYAs with enduring everyday pain. Public health nurses and school nurses should acknowledge their crucial roles in respecting AYAs' pain expressions and support them to use healthy coping strategies. Because of the increase in the use of OTC medications, they also have a role in informing AYAs about the side effects of such agents.

\section{Strengths and limitations}

The transparent and systematic methodological approach of this metasynthesis is a strength. One limitation could be the exclusion of studies not concerning everyday pain. On the other hand, this limitation is a strength as these AYAs' voices are seldom heard compared with those recruited from a clinical context. Synthesising data from nine qualitative studies with 184 participants provided rich qualitative data and in-depth knowledge about these AYAs' experiences. It could be a limitation that five of the nine studies focused on headache. On the other hand, it provides an important elaboration of previous quantitative studies describing headache as one of the most common pain conditions in adolescents. ${ }^{12}$ Another limitation is that all the studies were conducted in Western cultures. Studies from other contexts, or studies of exposed populations-for instance refugee AYAs—clearly would have helped to broaden the picture.

\section{CONCLUSION}

These AYAs experience of how pain influences everyday life, and their striving to find relief from pain by support from family, friends, professionals and the internet should be strongly respected. Public health nurses and other healthcare professionals encountering AYAs need to respect their pain experiences, and to support them in healthy coping strategies. Further studies on this issue are needed, especially research focusing on AYAs pain in exposed populations and AYAs from non-Western cultures.

\section{Author affiliations}

${ }^{1}$ Faculty of Health and Sport Sciences, University of Agder, Kristiansand, Vest-Agder, Norway

${ }^{2}$ Department of Pediatrics, Sørlandet Sykehus HF, Kristiansand, Norway
${ }^{3}$ Department of Clinical Medicine, Randers Regional Hospital, Randers, Midtjylland, Denmark

${ }^{4}$ Faculty of Nursing and Health Sciences, Nord University, Bodø, Norway

${ }^{5}$ Faculty of Health and Sport Sciences, University of Agder, Grimstad, Norway

${ }^{6}$ Department of Clinical Research, Sørlandet Sykehus HF, Kristiansand, Norway

${ }^{7}$ Faculty of Health Sciences, Oslo Metropolitan University, Oslo, Norway

Acknowledgements Librarian Ellen Sejersted at the University of Agder assisted in the development of the search strategy.

Collaborators Librarian Ellen Sejersted at the University of Agder contributed to the systematic literature search.

Contributors LF, MSL and KH designed the research project and developed the research plan. Librarian Ellen Sejersted at the University of Agder, LF, MSL and KH were responsible for the literature search, and LF, MSL, BJ and KH were responsible for the analysis. LF, MSL, BJ, TW, MH, ÅS, GR, SH and KH were involved in the screening and inclusion of studies, reviewed the manuscript, and contributed to the revision of the paper. All authors read and approved the final version of the paper.

Funding The authors have not declared a specific grant for this research from any funding agency in the public, commercial or not-for-profit sectors.

Competing interests None declared.

Patient and public involvement Patients and/or the public were not involved in the design, or conduct, or reporting, or dissemination plans of this research.

Patient consent for publication Not required.

Provenance and peer review Not commissioned; externally peer reviewed.

Data availability statement Data are available on reasonable request.

Open access This is an open access article distributed in accordance with the Creative Commons Attribution Non Commercial (CC BY-NC 4.0) license, which permits others to distribute, remix, adapt, build upon this work non-commercially, and license their derivative works on different terms, provided the original work is properly cited, appropriate credit is given, any changes made indicated, and the use is non-commercial. See: http://creativecommons.org/licenses/by-nc/4.0/.

ORCID iDs

Liv Fegran http://orcid.org/0000-0001-5470-8686

Mette Spliid Ludvigsen http://orcid.org/0000-0001-9439-380X

Magnhild Høie http://orcid.org/0000-0003-3131-5446

\section{REFERENCES}

1 Eccleston C, Palermo TM, de C Williams AC. Psychological therapies for the management of chronic and recurrent pain in children and adolescents. Cochrane database of systematic reviews 2012;12.

2 Gold JI, Yetwin AK, Mahrer NE, et al. Pediatric chronic pain and health-related quality of life. J Pediatr Nurs 2009;24:141-50.

3 Haraldstad K, Christophersen K-A, Eide H, et al. Predictors of healthrelated quality of life in a sample of children and adolescents: a school survey. J Clin Nurs 2011;20:3048-56.

4 Hoftun GB, Romundstad PR, Zwart J-A, et al. Chronic idiopathic pain in adolescence--high prevalence and disability: the young HUNT Study 2008. Pain 2011;152:2259-66.

5 Konijnenberg AY, Uiterwaal CSPM, Kimpen JLL, et al. Children with unexplained chronic pain: substantial impairment in everyday life. Arch Dis Child 2005;90:680-6.

6 Roth-Isigkeit A, Thyen U, Raspe HH. Reports of pain among German children and adolescents: an epidemiological study. Acta Paediatrica, International Journal of Paediatrics 2004;93:258-63.

7 van Dijk A, McGrath PA, Pickett W, et al. Pain and self-reported health in Canadian children. Pain Res Manag 2008;13:407-11.

8 Rabbitts JA, Holley AL, Groenewald CB, et al. Association between widespread pain scores and functional impairment and health-related quality of life in clinical samples of children. J Pain 2016;17:678-84.

9 Høie M, Haraldstad K, Rohde G, et al. How school nurses experience and understand everyday pain among adolescents. BMC Nurs 2017; $16: 53$.

10 Johannessen B, Hoie M, Haraldstad K. School nurses' and teachers' perceptions of pain in young immigrants living in Norway. International Journal of Migration, Health and Social Care 2020.

11 Mikkelsen HT, Haraldstad K, Helseth S, et al. Health-Related quality of life is strongly associated with self-efficacy, self-esteem, loneliness, and stress in 14-15-year-old adolescents: a crosssectional study. Health Qual Life Outcomes 2020;18:352. 
12 King S, Chambers CT, Huguet A, et al. The epidemiology of chronic pain in children and adolescents revisited: a systematic review. Pain 2011;152:2729-38.

13 Haraldstad K, Christophersen K-A, Helseth S. Health-Related quality of life and pain in children and adolescents: a school survey. BMC Pediatr 2017;17:174.

14 Brattberg G. Do pain problems in young school children persist into early adulthood? A 13-year follow-up. Eur J Pain 2004;8:187-99.

15 Perquin CW, Hazebroek-Kampschreur AAJM, Hunfeld JAM, et al. Pain in children and adolescents: a common experience. Pain 2000;87:51-8.

16 Petersen S, Brulin C, Bergström E. Recurrent pain symptoms in young schoolchildren are often multiple. Pain 2006;121:145-50.

17 McCaffery M, Beebe A. Making the best of inadequate analgesics. Nursing 1994;24:32C-32D.

18 Merskey $\mathrm{H}$. Logic, truth and language in concepts of pain. Qual Life Res 1994;3:S69-76.

19 Hoftun GB, Romundstad PR, Rygg M. Factors associated with adolescent chronic non-specific pain, chronic multisite pain, and chronic pain with high disability: the Young-HUNT study 2008. J Pain 2012;13:874-83.

20 Heathcote LC, Lau JYF, Mueller SC, et al. Child attention to pain and pain tolerance are dependent upon anxiety and attention control: an eye-tracking study. Eur J Pain 2017;21:250-63.

21 Meldrum ML, Tsao JC-I, Zeltzer LK. "I can't be what I want to be": children's narratives of chronic pain experiences and treatment outcomes. Pain Med 2009;10:1018-34.

22 Merlijn VPBM, Hunfeld JAM, van der Wouden JC, et al. Psychosocial factors associated with chronic pain in adolescents. Pain 2003;101:33-43.

23 Rathleff MS, Roos EM, Olesen JL, et al. High prevalence of daily and multi-site pain--a cross-sectional population-based study among 3000 Danish adolescents. BMC Pediatr 2013;13:191.

24 Tsao JCl, Meldrum M, Kim SC, et al. Anxiety sensitivity and health-related quality of life in children with chronic pain. J Pain 2007;8:814-23.

25 Incledon E, O'Connor M, Giallo R, et al. Child and family antecedents of pain during the transition to adolescence: a longitudinal population-based study. J Pain 2016;17:1174-82.

26 Liossi C, Noble G, Franck LS. How parents make sense of their young children's expressions of everyday pain: a qualitative analysis. Eur J Pain 2012;16:1166-75.

27 Reid K, Simmonds M, Verrier M, et al. Supporting teens with chronic pain to obtain high school credits: chronic pain 35 in Alberta. Children 2016;3:31.

28 Skarstein S, Lagerløv P, Kvarme LG, et al. High use of over-thecounter analgesic; possible warnings of reduced quality of life in adolescents - a qualitative study. BMC Nurs 2016;15:16.

29 Skarstein S, Rosvold EO, Helseth S, et al. High-Frequency use of over-the-counter analgesics among adolescents: reflections of an emerging difficult life, a cross-sectional study. Scand J Caring Sci 2014;28:49-56.

30 Hatchette JE, McGrath PJ, Murray M, et al. The role of peer communication in the socialization of adolescents' pain experiences: a qualitative investigation. BMC Pediatr 2008;8:2.

31 Holmström IK, Bastholm-Rahmner P, Bernsten C, et al. Swedish teenagers and over-the-counter analgesics - responsible, casual or careless use. Res Social Adm Pharm 2014;10:408-18.

32 Fegran L, Ludvigsen MS, Haraldstad K. Adolescents and young adults' experiences of living with everyday pain: a systematic review protocol of qualitative evidence. JBI Database System Rev Implement Rep 2014;12:116-26.

33 Sandelowski M, Barroso J, Voils $\mathrm{Cl}$. Using qualitative metasummary to synthesize qualitative and quantitative descriptive findings. Res Nurs Health 2007;30:99-111.

34 Moher D, Liberati A, Tetzlaff J, et al. Reprint--preferred reporting items for systematic reviews and meta-analyses: the PRISMA statement. Phys Ther 2009;89:873-80.

35 Walter SM. The experience of adolescents living with headache. Holist Nurs Pract 2017;31:280-9.

36 Skogvold L, Magnussen LH. Chronic tension-type headache and coping strategies in adolescents: a qualitative interview study. Physiother Res Int 2019;24:N.PAG.

37 Critical appraisal tools: Joanna Briggs Institute, 2018. Available: http://joannabriggs.org/research/critical-appraisal-tools.html [Accessed January 25th 2018].

38 Henderson EM, Keogh E, Eccleston C. Why go online when you have pain? A qualitative analysis of teenagers' use of the Internet for pain management advice. Child Care Health Dev 2014;40:572-9.
39 Kernick D, Reinhold D, Stone C. A qualitative study of headache in school children. British Journal of School Nursing 2011;6:337-41.

40 Hansen DL, Hansen EH, Holstein BE. Young women's use of medicines: autonomy and positioning in relation to family and peer norms. Health 2009;13:467-85.

41 Hatchette JE, McGrath PJ, Murray M. Maternal influences in adolescents' pain self-management: a qualitative investigation. Vulnerable Children and Youth Studies 2006;1:159-69.

42 Henderson EM, Eccleston C. An online adolescent message board discussion about the Internet: use for pain. J Child Health Care 2015;19:412-8

43 Huguet A, Stinson J, Mackay B, et al. Bringing psychosocial support to headache sufferers using information and communication technology: lessons learned from asking potential users what they want. Pain Res Manag 2014;19:e1-8.

44 NVivo 12: QSR International;. 2018 [Qualitative computer software package], 2018. Available: https://www.qsrinternational.com/nvivoqualitative-data-analysis-software/home

45 Lindseth A, Norberg A. A phenomenological hermeneutical method for researching lived experience. Scand J Caring Sci 2004;18:145-53.

46 Graneheim UH, Lindgren B-M, Lundman B. Methodological challenges in qualitative content analysis: a discussion paper. Nurse Educ Today 2017;56:29-34.

47 Graneheim UH, Lundman B. Qualitative content analysis in nursing research: concepts, procedures and measures to achieve trustworthiness. Nurse Educ Today 2004;24:105-12.

48 Ahlqwist A, Sällfors C. Experiences of low back pain in adolescents in relation to physiotherapy intervention. Int J Qual Stud Health Wellbeing 2012;7:15471.

49 Hansen DL, Hansen EH, Holstein BE. Using analgesics as tools: young women's treatment for headache. Qual Health Res 2008;18:234-43.

50 Hansen DL, Holstein BE, Hansen EH. "I'd Rather Not Take it, but...": Young Women's Perceptions of Medicines. Qual Health Res 2009;19:829-39.

51 Raja SN, Carr DB, Cohen M, et al. The revised international association for the study of pain definition of pain: concepts, challenges, and compromises. Pain 2020;161:1976-82.

52 Rohde G-E, Westergren T, Haraldstad K. Teachers' experiences of adolescents' pain in everyday life: a qualitative study. BMJ Open 2015;5:7 http://dx.doi.org

53 Giota J, Gustafsson J-E. Perceived demands of schooling, stress and mental health: changes from grade 6 to grade 9 as a function of gender and cognitive ability. Stress Health 2017;33:253-66.

54 Castarlenas E, Vega Rdela, Tomé-Pires C, et al. Student expectations of peer and teacher reactions to students with chronic pain: implications for improving pain-related functioning. Clin J Pain 2015;31:992-7.

55 Slater H, Jordan JE, Chua J, et al. Young people's experiences of persistent musculoskeletal pain, needs, gaps and perceptions about the role of digital technologies to support their co-care: a qualitative study. BMJ Open 2016;6:e014007.

56 Sørensen K, Christiansen B. Adolescents' experience of complex persistent pain. Scand J Pain 2017:15:106-12.

57 Gorodzinsky AY, Hainsworth KR, Weisman SJ. School functioning and chronic pain: a review of methods and measures. J Pediatr Psychol 2011;36:991-1002.

58 Fisher E, Law E, Dudeney J, et al. Psychological therapies (remotely delivered) for the management of chronic and recurrent pain in children and adolescents. Cochrane Database Syst Rev 2019;4:CD011118.

59 Tse MMY, Tang A, Budnick A, et al. Pain and pain management among university students: online survey and web-based education. Cyberpsychol Behav Soc Netw 2017;20:305-13.

60 Hundert AS, Huguet A, McGrath PJ. Commercially available mobile phone headache diary apps: a systematic review. Journal of Medical Internet Research 2014;16:e36.

61 Suris J-C, Michaud P-A, Viner R. The adolescent with a chronic condition. Part I: developmental issues. Arch Dis Child 2004;89:938-42.

62 Fegran L, Hall EOC, Uhrenfeldt L, et al. Adolescents' and young adults' transition experiences when transferring from paediatric to adult care: a qualitative metasynthesis. Int J Nurs Stud 2014;51:123-35.

63 Skarstein S, Lagerløv P, Helseth S, et al. How do parents influence their adolescents' use of over-the-counter analgesics: a review of the current literature. J Clin Nurs 2019;28:1451-64. 